\title{
CONTRIBUIÇÃO DOS DEPÓSITOS HEPÁTICOS DE IMUNOGLOBULINA A NO DIAGNÓSTICO DA HEPATOPATIA ALCOÓLICA $^{+}$
}

\author{
Luiz Edmundo MAZZOLENI", Maria Isabel Albano EDELWEISS ${ }^{* *}$, \\ Carlos KUPSKI $^{* * *}$, Sérgio Gabriel Silva de BARROS ${ }^{* * * *}$ e Carlos Luiz REICHEL ${ }^{* * * * *}$
}

RESUMO - Racional - A doença hepática alcoólica é enfermidade grave e freqüente e o seu diagnóstico nem sempre é fácil. Objetivo - Avaliar a contribuição de depósitos de imunoglobulina A (IgA) nos sinusóides hepáticos para o diagnóstico da hepatopatia alcoólica. Casuística e Método - Foi estudada a presença de imunoglobulina A por imunofluorescência direta em 59 pacientes submetidos a punção biopsia hepática, indicada por alterações clínicas ou laboratoriais sugestivas de hepatopatia crônica. Resultados - Constatou-se deposição significativa de imunoglobulina A hepática em alcoolistas, em comparação com os não-alcoolistas, com sensibilidade de 76\% (IC95\%: 54,5-89,8) e especificidade de 73,5\% (IC95\%. 55,3-86,5). Em indivíduos que apresentavam só o álcool como agente etiológico da hepatopatia, comparados com o grupo de portadores dos virus B e C, os resultados foram ainda mais significativos, com sensibilidade de 85, 7\% (IC95\%: 56,297,5) e especificidade de 89,5\% (IC95\%: 65,5\%-98,2\%). Conclusão - A deposição de imunoglobulina A nos sinusóides hepáticos apresenta sensibilidade e especificidade para o diagnóstico da lesão hepática induzida pelo álcool. Esse recurso pode ser particularmente útil quando a histologia convencional não consegue definir a causa específica da alteração encontrada.

DESCRITORES - Hepatopatias alcoólicas, diagnóstico. IGA. Hepatopatias alcoólicas, patologia. Biopsia.

Trabalho realizado no Hospital São Lucas da Pontifícia Universidade Católica do Rio Grande do Sul (PUCRS) e Hospital de Clínicas de Porto Alegre da Universidade Federal do Rio Grande do Sul (UFGRS) para obtenção do título de Mestre no Programa de Pós-Graduação em Medicina: Gastroenterologia da Faculdade de Medicina da UFRGS.

* Professor Assistente da Faculdade de Medicina da UFRGS e Faculdade de Medicina da PUCRS. Médico do Serviço de Gastroenterologia do Hospital de Clínicas de Porto Alegre.

** Professora Adjunta, Departamento de Patologia da Faculdade de Medicina da UFRGS.

*** Professor Assistente, Departamento de Medicina Interna da Faculdade de Medicina da PUCRS.

**** Professor Adjunto, Departamento de Medicina Interna. Coordenador da Comissão de Pós-Graduação da Faculdade de Medicina da UFRGS. Médico do Serviço de Gastroenterologia do Hospital de Clínicas de Porto Alegre.

***** Professor Titular de Anatomia Patológica da Faculdade de Medicina da PUCRS.

Endereço para correspondência: Dr. Luiz Edmundo Mazzoleni - Programa de Pós-Graduação em Medicina: Gastroenterologia - Rua Ramiro Barcelos, $2400,2^{\circ}$ andar - Santana -90035-001 - Porto Alegre, RS. e-mail: mazzoleni@pro.via-rs.com.br 


\section{INTRODUÇÃO}

A confirmação da doença hepática alcoólica (DHA) é difícil. Dados de anamnese sobre a real ingestão de bebidas alcoólicas são pouco confiáveis e nenhum dos testes laboratoriais pode indicar, inequivocamente, o abuso de álcool. Na histologia, o dano hepático causado pelo álcool pode incluir um espectro variável de alterações como esteatose, esteatohepatite e cirrose, mas essas características não são patognomônicas ${ }^{(1,3,10)}$.

Em 1965, LEE et al. ${ }^{(6)}$ constataram que, na doença hepática associada ao álcool, havia aumento sérico da imunoglobulina A (IgA), mas não em hepatites virais. Posteriormente, SWERDLOW et al. ${ }^{(10)}$ demonstraram que a IgA depositava-se no fígado em casos de doença crônica causada por álcool. A imunofluorescência direta tem demonstrado depósitos de IgA nos sinusóides hepáticos de alcoolistas, o que não ocorre em outras formas de hepatopatia crônica ${ }^{(3,7,10)}$.

Van de WIEL et al. ${ }^{(11)}$, em 1988, publicaram estudo demonstrando alta sensibilidade e especificidade do depósito de IgA em doença hepática crônica alcoólica e sugeriram incluir, no exame histológico hepático convencional, a avaliação imunohistológica para aumentar o acerto diagnóstico.

A causa desse depósito não está bem definida, todavia pode decorrer de resposta imunológica humoral a metabólitos do álcool nos hepatócitos, especialmente aos chamados adutos do acetaldeído ${ }^{(4,12)}$. Esses depósitos podem ter papel na patogênese do dano hepático do álcool ou refletir os seus efeitos ${ }^{(2)}$.

O presente estudo avaliou a contribuição do depósito de IgA nos sinusóides hepáticos, como marcador de DHA.

\section{CASUÍ́STICA E MÉTODO}

Foi realizado estudo transversal que avaliou a prevalência do depósito de IgA em hepatopatias de várias etiologias, cujo desfecho foi o diagnóstico da hepatopatia alcoólica pela imunofluorescência da IgA nos sinusóides hepáticos.

O estudo foi prospectivo e a amostra foi constituída por 65 pacientes consecutivos, 43 do sexo masculino e 22 do sexo feminino, oriundos dos ambulatórios ou das enfermarias do Hospital São Lucas da Pontifícia Universidade Católica do Rio Grande do Sul (PUCRS), que apresentaram sinais clínicos e/ou alterações laboratoriais de doenças hepáticas crônicas, com indicação para realizarem biopsia hepática no período de maio de 1993 até setembro de 1994. A biopsia hepática foi realizada após os pacientes assinarem consentimento informado sobre o procedimento e os objetivos da pesquisa. O estudo foi aprovado pelo Comitê de Ética em Pesquisa do Hospital São Lucas da PUCRS.

Os pacientes foram divididos em dois grupos: alcoolistas (A) e não-alcoolistas (NA).

O grupo A consistia de pacientes que faziam consumo de álcool em média diária igual ou superior a $80 \mathrm{~g} /$ dia para homens e $50 \mathrm{~g} / \mathrm{dia}$ para mulheres, em período igual ou superior a 5 anos. $\mathrm{O}$ instrumento utilizado para definir e quantificar a ingesta alcoólica foi a aplicação de questionários com adequada técnica semiológica, em todos os pacientes pelo mesmo investigador. Para obtenção do equivalente em gramas de álcool puro para os diversos tipos de bebidas, foi considerada a concentração média de etanol nas bebidas alcoólicas. Foram consideradas as seguintes equivalências: $500 \mathrm{~mL}$ de cerveja $=20 \mathrm{~g}$; $100 \mathrm{~mL}$ de vinho $=10 \mathrm{~g}$ e $50 \mathrm{~mL}$ de destilados $=20 \mathrm{~g}$ de etanol.

O grupo NA era constituído por pacientes sem ingestão crônica abusiva de álcool e com suspeita de doença hepática associada a vírus, drogas ou doenças sistêmicas.

Todos os pacientes incluídos realizaram a dosagem sérica do HBsAg pelo teste ELISA e do Anti-VHC pelo teste ELISA II (Abbott Laboratórios).

As drogas utilizadas pelos pacientes e suas doenças sistêmicas foram consideradas no estudo quando apresentassem potencial hepatotóxico.

Os fragmentos de fígado obtidos com a biopsia eram divididos, sendo que aproximadamente a metade dos mesmos era fixada em formol e encaminhada para estudo histológico convencional e a outra metade era transportada em solução fisiológica em período de tempo de até 10 minutos, sendo então congelada em nitrogênio líquido e, após, armazenada para estudo por imunofluorescência direta. No processamento, o tecido era submetido aos seguintes passos:

- corte em micrótomo criostato, a $20^{\circ} \mathrm{C}$, na espessura de 6 micrômetros;

- fixação das lâminas com os cortes em acetona gelada;

- três lavagens em solução de tampão fosfato PBS (pH 7,2);

- incubação com anticorpos anti-IgA (teste) e anti-albumina (controle) humanas conjugadas à fluoresceína, produzidas em coelho, marca Bayer, diluídas em solução de tampão fosfato pH 7,2 (PBS) de 1/20, durante 30 minutos em câmara úmida;

- lavagem em três banhos de solução de PBS (pH 7,2);

- montagem com lamínula em meio de glicerina;

- observação ao microscópio de epifluorescência, nos aumentos de 200 e 400 vezes; e

- após, as lâminas eram examinadas por médica patologista que desconhecia os diagnósticos anatomopatológicos e os casos clínicos, emitindo laudo final da biopsia.

Os resultados dos depósitos de IgA hepática foram classificados em três tipos: ausência de depósito (AD), depósito hepático com padrão descontínuo (PD), que é interrompido ao longo dos sinusóides, e padrão contínuo (PC), em que a IgA é ininterrupta. O presente estudo classificou, para fins de análise dos resultados, os depósitos de IgA em dois grupos: $1^{\circ}$ ) presença de IgA hepática (P-IgA) - neste, estão inclusos os tipos de depósito "descontínuo" e "contínuo"; e $2^{\circ}$ ) ausência de IgA hepática (A-IgA).

O nível de significância determinado em todos os testes foi de 5\%. 


\section{RESULTADOS}

\section{Características da Amostra}

A descrição da amostra dos pacientes é apresentada na Tabela 1.

Como o estudo foi prospectivo e os grupos estudados não foram pareados, mas divididos apenas pela variável básica, que foi o uso abusivo do álcool, existem diferenças entre os grupos pelas características demográficas próprias dos pacientes A e NA. Uma diferença estatisticamente significativa ocorreu na idade média dos grupos (maior nos A), mas ficou constatado que, quando a amostra foi estratificada em duas faixas de idade, uma dos 20-40 anos e a outra dos 41-70 anos, ficaram mantidas as diferenças estatisticamente significativas entre $o$ grupo de A e de NA quanto ao depósito de IgA hepática. A literatura não tem citações considerando a idade como um fator envolvido no depósito hepático de IgA. Quanto aos outros dados (vírus, drogas), foi realizada uma "depuração" desses fatores de confusão, ao serem separados os grupos em subgrupos, agredidos apenas pelo álcool ou pelos vírus.

Do total de 65 pacientes que realizaram biopsia hepática, 6 foram excluídos do estudo por perda do material da imunofluorescência.

\section{Alterações Histológicas}

A Tabela 2 apresenta as alterações histológicas encontradas na amostra de pacientes.

A alteração mais freqüentemente observada nos grupos estudados foi a hepatite crônica ativa (HCA), especialmente no grupo NA ( $\mathrm{n}=$ 17) e em menor freqüência no grupo $A(n=3)$. No período do estudo, ainda estava em uso a classificação das hepatites crônicas em "ativa" e "persistente". A HCA representou 33,9\% do total dos achados histológicos da amostra (20 de 59 pacientes).
A esteatose foi a segunda alteração histológica mais observada ( 9 A e 6 NA), em um total de 15 pacientes, representando $25,4 \%$ da amostra.

\section{Depósitos de IgA hepática A x NA}

Para fins de análise dos depósitos hepáticos de $\operatorname{IgA}$, os pacientes foram classificados em dois grupos (A e NA) (Tabela 3) e em dois subgrupos (Tabela 4), caso apresentassem só o álcool como agente etiológico (AA), ou se apenas foram encontrados os vírus $\mathrm{B}$ e $\mathrm{C}$, sem outros agressores associados (VV).

Os resultados dos depósitos de IgA hepática são apresentados nas Tabelas 3 e 4.

\section{Depósitos de IgA Hepática x Histologia}

As Tabelas 5 e 6 demonstram os depósitos de IgA nas alterações hepáticas mais freqüentemente observadas na histologia convencional.

O número de pacientes nas outras alterações histológicas (Tabela 2) foi insuficiente para análise estatística do depósito de IgA hepática.

\section{DISCUSSÃO}

O primeiro passo para o diagnóstico da DHA costuma ser a definição, pela história clínica, do abuso alcoólico. No entanto, nem sempre a anamnese consegue definir essa situação, pois os pacientes, com grande freqüência, omitem ou diminuem as quantidades de álcool que ingerem. Embora a grande maioria dos estudos classifique seus pacientes com base nos dados de anamnese, ela é um "padrão referencial" pouco confiável nesta patologia. Os marcadores bioquímicos, como o VCM e a gama-GT, apresentam limitada capacidade de estabelecer o diagnóstico de doença hepática alcoólica ${ }^{(11)}$, e não foram analisados no presente estudo.

TABELA 1 - Descrição da amostra

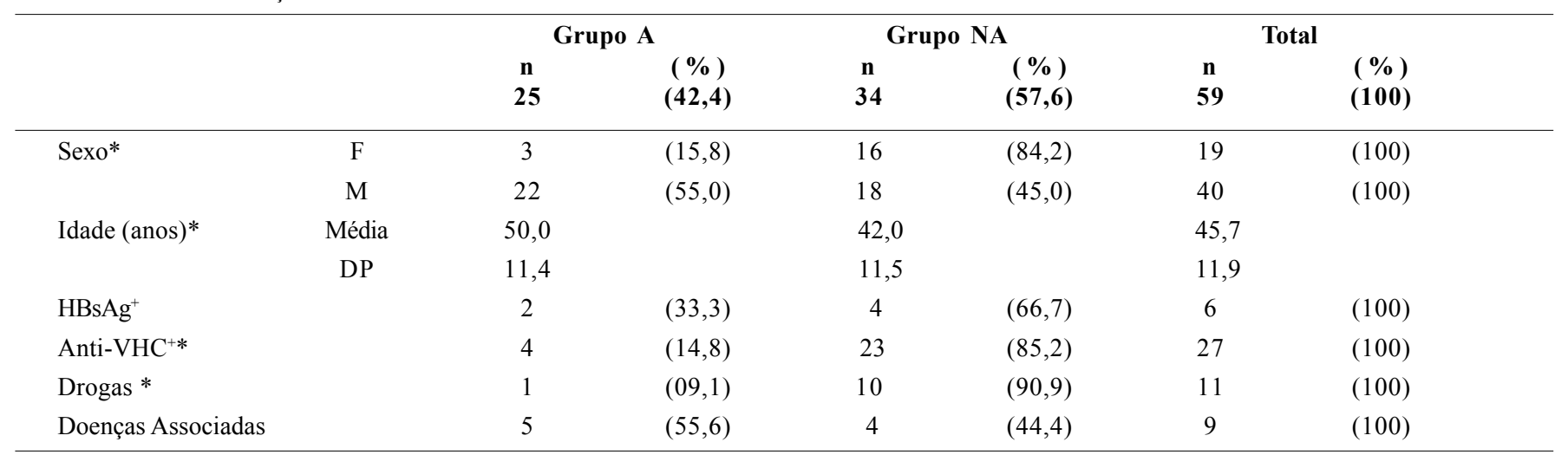

$* P<0,05$ entre os grupos A e NA

$\mathrm{A}=$ alcoolista $; \mathrm{NA}=$ não-alcoolista $\mathrm{F}=$ feminino $\mathrm{M}=$ masculino; $\mathrm{DP}=$ desvio padrão 
Mazzoleni LE, Edelweiss MIA, Kupski C, Barros SGS, Reichel CL. Contribuição dos depósitos hepáticos de imunoglobulina A no diagnóstico da hepatopatia alcoólica

TABELA 2 - Alterações histológicas

\begin{tabular}{lcccc}
\hline $\mathbf{n}$ & Histologia & A & NA & \% \\
\hline 20 & hepatite crônica ativa & 3 & 17 & 33,9 \\
15 & esteatose & 9 & 6 & 25,4 \\
7 & cirrose & 5 & 2 & 11,9 \\
2 & hepatite aguda & - & 2 & 3,3 \\
7 & normais & 3 & 4 & 11,9 \\
8 & inespecíficas & 5 & 3 & 13,5 \\
59 & total & 25 & 34 & 100 \\
\hline
\end{tabular}

TABELA 3 - Depósito de IgA nos grupos A x NA

\begin{tabular}{lccccc}
\hline & \multicolumn{2}{c}{$\mathbf{A}=\mathbf{2 5 *}$} & \multicolumn{2}{c}{ NA = 34* } \\
\hline $\operatorname{IgA}$ & $\mathrm{n}$ & $\mathrm{n}$ & $(\%)$ & $\mathrm{n}$ & $(\%)$ \\
$\mathrm{P}-\operatorname{IgA}=$ & 28 & 19 & $(76,0)$ & 9 & $(26,5)$ \\
$\mathrm{A}-\operatorname{IgA}=$ & 31 & 6 & $(24,0)$ & 25 & $(73,5)$
\end{tabular}

Sensibilidade: 76 (IC95\%: 54,5-89,8)

Especificidade: 73,5 (IC95\%: 55,3-86,5)

$* P<0,001$

$\mathrm{P}-\operatorname{IgA}=$ presença de $\operatorname{IgA}$ hepática A-IgA = ausência de IgA hepática

TABELA 4 - Depósito de IgA nos subgrupos AA x VV

\begin{tabular}{lccccc}
\hline & \multicolumn{2}{c}{$\mathbf{A A}=\mathbf{1 4}$} & \multicolumn{2}{c}{$\mathbf{V V}=\mathbf{1 9 *}$} \\
\hline $\mathrm{IgA}$ & $\mathrm{n}$ & $\mathrm{n}$ & $(\%)$ & $\mathrm{n}$ & $(\%)$ \\
$\mathrm{P}-\operatorname{IgA}=$ & 14 & 12 & $(85,7)$ & 2 & $(10,5)$ \\
$\mathrm{A}-\operatorname{IgA}=$ & 19 & 2 & $(14,3)$ & 17 & $(89,5)$ \\
\hline
\end{tabular}

Sensibilidade: 85,7 (IC95\%: 56,2-97,5)

Especificidade: 89,5 (IC95\%: 65,5-98,2)

$* P<0,001$

P-IgA = presença de IgA hepática; A-IgA = ausência de IgA hepática

$\mathrm{AA}=$ somente álcool; $\mathrm{VV}=$ somente vírus

TABELA 5 - Hepatite crônica ativa e IgA hepática $(\mathrm{n}=20)$

\begin{tabular}{ccccc} 
& \multicolumn{2}{c}{ Grupos* $^{2}$} & \multicolumn{2}{c}{ Subgrupos } \\
\hline & $\mathrm{A}$ & $\mathrm{NA}$ & $\mathrm{AA}$ & $\mathrm{VV}$ \\
$\mathrm{P}-\mathrm{IgA}$ & $\mathrm{n}$ & $\mathrm{n}$ & $\mathrm{n}$ & $\mathrm{n}$ \\
$\mathrm{A}-\operatorname{IgA}$ & 3 & 4 & 1 & 1 \\
\hline & $\varnothing$ & 13 & $\varnothing$ & 11 \\
\hline
\end{tabular}

$* P<0,05$ (Teste exato de Fisher bicaudal)

$\mathrm{P}-\operatorname{IgA}=$ presença de IgA hepática; A-IgA = ausência de IgA hepática;

$\mathrm{A}=$ alcoolistas; $\mathrm{NA}=$ não-alcoolistas;

$\mathrm{AA}=$ somente álcool; $\mathrm{VV}=$ somente vírus
TABELA 6 - Esteatose e IgA hepática $(n=15)$

\begin{tabular}{|c|c|c|c|c|}
\hline & \multicolumn{2}{|c|}{ Grupos* } & \multicolumn{2}{|c|}{ Subgrupos* } \\
\hline & $\mathrm{A}$ & NA & $\mathrm{AA}$ & W \\
\hline & $\mathrm{n}$ & $\mathrm{n}$ & $\mathrm{n}$ & $\mathrm{n}$ \\
\hline P-IgA & 8 & 1 & 6 & $\varnothing$ \\
\hline A-IgA & 1 & 5 & $\varnothing$ & 3 \\
\hline $\begin{array}{l}P<0,05 \\
P-\operatorname{IgA}=\text { pre } \\
\mathrm{A}=\text { alcooli } \\
\mathrm{AA}=\text { some }\end{array}$ & não & $\begin{array}{l}\text { A-Ig } \\
\text { tas; } \\
\text { te vír }\end{array}$ & a de Is & \\
\hline
\end{tabular}

Para uma completa interpretação dos resultados obtidos neste estudo, é fundamental que três importantes fatores de confusão sejam lembrados:

1) A omissão de informações sobre a ingesta alcoólica pode ter classificado A como sendo NA: presença de IgA - falso-positivo.

2) Alguns pacientes podem ter apresentado lesões hepáticas com doses alcoólicas abaixo das consideradas hepatotóxicas neste estudo $^{(9)}$, por suscetibilidades individuais ao álcool: presença de IgA - falso-positivo.

3) Alguns pacientes que usavam doses alcoólicas definidas como hepatotóxicas e que foram corretamente definidos como A, podem ter "resistência" natural ao dano hepático pelo álcool e não apresentarem hepatopatia: ausência de $\operatorname{IgA}$ - falso-negativo.

Por critérios estabelecidos no projeto da pesquisa, o objetivo básico foi definir se a IgA estava ou não presente no fígado, não sendo avaliado o tipo da deposição (contínua ou descontínua). Os resultados foram muito semelhantes aos de outras publicações, quando os padrões contínuos da literatura são comparados com os positivos do presente estudo. Isso demonstra que os padrões descontínuos da literatura foram considerados como negativos para IgA neste estudo, da mesma forma como o foram em outros estudos ${ }^{(5)}$. No presente estudo, os depósitos de IgA hepática foram significativamente mais freqüentes no grupo dos A (76\%), do que no grupo dos NA (26.5\%). SWERDLOW et al. ${ }^{(10)}$ encontraram depósito contínuo de IgA em 50 de 64 (78\%) biopsias hepáticas de A e este achado ocorreu em apenas 3 de 72 (4.16\%) NA. Van de WIEL et al. ${ }^{(11)}$ obtiveram especificidade de 0.91 e sensibilidade de 0.75 na presença de $\operatorname{IgA}$, em um padrão de depósito contínuo no fígado de etilistas, e consideraram este o melhor teste para sugerir abuso alcoólico como etiologia da doença hepática alcoólica.

Além da análise dos grupos dos A e NA, os pacientes deste estudo foram divididos em subgrupos, em que apenas o álcool (AA) ou somente os vírus B e C (VV) estavam presentes. A especificidade $\mathrm{e}$ sensibilidade do depósito de IgA hepática foram ainda maiores nesses subgrupos, como demonstrado na Tabela 4. No subgrupo AA, em que o álcool foi o único agressor hepático, apenas 2 pacientes de um total 
de $14(14,3 \%)$ não apresentaram presença de IgA no fígado, o que poderia ser explicado, nesses dois pacientes que foram exceção, pela "resistência" que certos indivíduos apresentam ao dano hepático do álcool. Por sua vez, em pacientes em que apenas os vírus $\mathrm{B}$ e $\mathrm{C}$ foram identificados como agressores hepáticos (subgrupo VV), só 2 de 19 pacientes $(10,5 \%)$ apresentaram presença de depósito de IgA. Considerando como alta a chance de erro diagnóstico do alcoolismo apenas pelos dados de anamnese, é aceitável pensar que os dois pacientes positivos para $\operatorname{IgA}$ hepática, nesse subgrupo, eram etilistas que omitiram esta informação no questionário clínico (falha na avaliação clínica).

A análise dos pacientes com hepatite crônica ativa apresentados na Tabela 5, mostra que a maioria deles não apresentou depósitos hepáticos de IgA. No subgrupo VV apenas com a presença dos vírus B ou C, o depósito de IgA foi ainda mais infreqüente, sugerindo que, quando existir presença de IgA hepática nesse achado histológico, deve ser levantada a suspeita de concomitância, ou predomínio, de agressão alcoólica. Estes resultados são comparáveis aos da literatura. SWERDLOW et al. ${ }^{(10)}$ encontraram depósitos de IgA em 12 de 15 pacientes com hepatite crônica de A e somente em 1 de 15 casos de HCA em NA. No presente estudo, apenas um paciente apresentou IgA no fígado, entre 12 pacientes com hepatite crônica ativa no subgrupo portador apenas de agressão viral (Tabela 5). No grupo dos $\mathrm{A}$, os três pacientes com hepatite crônica ativa foram positivos para IgA hepática e, apesar de dois apresentarem também o vírus $\mathrm{C}$, o álcool deve ser considerado como provável agente causal das alterações hepáticas.

Nos pacientes A com esteatose hepática (Tabela 6), houve presença de depósito de IgA hepática na maioria deles (oito de nove pacientes). Nos NA com esteatose, apenas um de seis pacientes foi positivo para IgA hepática. Nos subgrupos, os resultados foram ainda mais marcantes, com todos os pacientes no subgrupo "só álcool" apresentando IgA hepática, e todos "só vírus" com ausência dessa deposição. No subgrupo "só vírus", foi observada a presença de três pacientes com esteatose, dois dos quais com Anti- $\mathrm{VHC}^{+}$e um com $\mathrm{HBs} \mathrm{Ag}^{+}$, nenhum deles apresentou IgA hepática, sugerindo presença de esteatose secundária apenas à agressão viral. A presença de esteatose na histologia hepática de pacientes com vírus é um achado comum ${ }^{(8)} \mathrm{e}$, nesses casos, a ausência da IgA hepática sugere lesão hepática viral, enquanto que a sua presença indica lesão alcoólica.

Um dado curioso é a comparação da IgA hepática entre os sexos. No grupo supostamente NA, 8 de 16 mulheres apresentaram IgA hepática, ao passo que apenas 1 de 18 homens NA apresentou esses achados. Esses resultados demonstraram maior positividade da IgA hepática nas mulheres, classificadas como NA, do que nos homens NA. A anamnese é pouco sensível na determinação do alcoolismo e, embora sem dados específicos, observa-se na prática assistencial que as mulheres têm maior dificuldade de declarar abusos alcoólicos, o que permitiria ter classificado mais mulheres A no grupo NA, do que homens. Portanto, a falha seria na história clínica que, mesmo corretamente aplicada, não teria detectado o etilismo pela omissão de informações. Outra possibilidade seria a maior suscetibilidade das mulheres ao álcool, que com doses menores do que as $50 \mathrm{~g} / \mathrm{dia}$ (grupo NA) poderiam apresentar dano hepático e, conseqüentemente, depositar IgA no fígado. Contra essa hipótese, o mesmo poderia estar acontecendo no grupo dos homens, que também poderiam sofrer lesões hepáticas com ingestas alcoólicas menores do que $80 \mathrm{~g} / \mathrm{dia}$. Esse achado, portanto, sugere que as mulheres tenham ocultado o verdadeiro consumo alcoólico.

A opinião de vários autores da literatura ${ }^{(3,10,11)}$, assim como a dos autores deste estudo, é que a deposição de IgA hepática pode ser mais confiável do que a história clínica, para definir lesão hepática alcoólica. Portanto, no caso de ser encontrada positividade desse marcador no fígado com anamnese negativa para abuso alcoólico, sugere-se confirmação detalhada da história clínica, enfatizando a importância desse achado ao próprio paciente, além de buscar informações sobre a ingesta alcoólica do paciente com outras fontes (familiares, amigos, etc.). Na situação inversa, ou seja, em pacientes hepatopatas com anamnese positiva para abuso alcoólico, mas com IgA negativa no tecido hepático, deve ser cogitada a possibilidade de não haver suscetibilidade hepática ao álcool, devendo ser pesquisados outros agressores, além do álcool.

Embora a eficácia diagnóstica do depósito hepático de IgA no diagnóstico diferencial das hepatopatias aparentemente não seja a ideal, o presente estudo e a literatura demonstram que a sensibilidade deste teste é superior à anamnese (utilizada para definir alcoolismo e lesão hepática alcoólica na maioria dos estudos). Além disso, alguns pesquisadores sugerem que a presença de $\operatorname{IgA}$ hepática seria o mais precoce e sensível indicativo de dano hepático pelo álcool ${ }^{(11)}$. Portanto, resultados falso-positivos de IgA hepática podem ser falha da anamnese, que não diagnosticou o alcoolismo. E, como a maioria dos A crônicos não desenvolve doença hepática alcoólica, a ausência de IgA no fígado poderá estar indicando ausência de dano hepático, apesar do abuso alcoólico, e não resultados falso-negativos. A verdadeira eficácia diagnóstica do depósito hepático de IgA somente poderá ser adequadamente avaliada, quando houver um "teste ouro" confiável do abuso alcoólico.

Em determinados subgrupos de alterações histológicas hepáticas, como nas hepatites crônicas e na esteatose, a IgA no fígado demonstrou grande sensibilidade e especificidade, sugerindo fortemente a etiologia da alteração hepática. Em casos de agressores simultâneos, como álcool e vírus, mesmo quando a histologia convencional não consegue definir qual dos agressores está lesando o fígado, a pesquisa da IgA hepática tem importante papel no diagnóstico diferencial.

Concluindo, a pesquisa da IgA hepática é método com boa sensibilidade e especificidade no diagnóstico da lesão hepática pelo 
álcool. Esse recurso pode ser particularmente útil em situações em que ocorra falha no diagnóstico clínico de abuso alcoólico, em pacientes simultaneamente agredidos por mais do que um agente etiológico e quando a histologia convencional não consegue definir a causa da alteração encontrada, com especial importância nos pacientes que apresentam esteatose e hepatites crônicas, quando, possivelmente, só o depósito de IgA possa definir a causa das alterações.

Mazzoleni LE, Edelweiss MIA, Kupski C, Barros SGS, Reichel CL. Contribution of hepatic immunoglobulin A deposits to the diagnosis of alcoholic hepatopathy. Arq Gastroenterol 2001;38(3):162-167.

ABSTRACT - Background - Alcoholic hepatic disease is a severe and frequent disease and its diagnosis is not always an easy task. Aim - To assess the contribution of immunoglobulin A (IgA) in the hepatic sinusoids for diagnosis of alcoholic hepatopathy. Patients and methodThe presence of IgA was studied through direct immunofluorescence in 59 patients submitted to hepatic needle biopsy, indicated by clinical or in vitro changes suggestive of chronic hepatopathy. Results - A significant deposition of IgA was found in alcoholic patients as compared to non-alcoholic patients, with 76\% sensitivity (95\%CI: 54.5-89.8) and 73.5\% specificity (95\%CI: 55.3-86.5). In individuals who present only alcohol as the etiological agent of hepatopathy, compared with the subgroup of $B$ or $C$ virus carriers, the results were even more significant, with $85.7 \%$ sensitivity (95\%CI: 56.2-97.5) and 89.5\% specificity (95\% CI: 65.5-98.2). Conclusion - The deposition of IgA in the hepatic sinusoids presents sensitivity and specificity for the diagnosis of an alcohol-induced hepatic lesion. This resource can be particularly useful when conventional histology can not be define a specific cause for the change found.

HEADINGS-Alcoholic liver disease, diagnosis. IGA. Alcoholic liver disease, pathology. Biopsy.

\section{REFERÊNCIAS BIBLIOGRÁFICAS}

1. Alcoholic liver disease: morphological manifestations. Review by an international group. Lancet 1981;1:707-11.

2. Gluud C, Christoffersen P, Eriksen J, Wantzin, Knudsen BB, The Copenhagen Study Group For Liver Disease. Influence of ethanol on development of hyperplastic nodules in alcoholic men with micronodular cirrhosis. Gastroenterology 1987;93:256-60.

3. Goldin RD, Cattle S, Boylston AW. IgA deposition in alcoholic liver disease. J Clin Pathol 1986;39:1181-5.

4. Israel Y, Orrego H, Niemelä O. Immune responses to alcohol metabolites: pathogenic and diagnostic implications. Semin Liver Dis 1988;8:81-90.

5. Kater L, Jobsis AC, Baart De La Faille-Kuyper EH, Vogten AJM, Grijm R. Alcoholic hepatic disease. Specificity of IgA deposits in liver. Am J Clin Pathol 1979;71:51.

6. Lee RG. Immunoglobulins in viral hepatitis and active alcohol liver disease. Lancet $1965 ; 1042$.
7. Mendenhall CL. Alcoholic hepatitis. In: Schiff L, Schiff ER, editors. Diseases of the liver. 7. ed. Philadelphia: JB Lippincott; 1993. p.856-74.

8. Sleisenger MH, Feldman M, Scharschmidt BF. Sleisenger \& Fordtran's Gastrointestinal and Liver Disease: Pathophysiology/Diagnosis/Management. 6. ed. Philadelphia: WB Saunders; 1997. p.1199-214.

9. Sorensen TI, Orholm M, Bentsen KD, Hoybye G, Eghoje K, Christoffersen P. Prospective evaluation of alcohol abuse and alcoholic liver injury in men as predictors of development of cirrhosis. Lancet 1984;2:241-4.

10. Swerdlow MA, Chowdhury LN, Horn T. Patterns of IgA deposition in liver tissues in alcoholic liver disease. Am J Clin Pathol 1982;77:259-66.

11. Van De Wiel A, Van Hattum J, Schuurman HJ, Kater L. Immunoglobulin A in the diagnosis of alcoholic liver disease. Gastroenterology 1988;94:457-62.

12. Worrall S, De Jersey J, Shanley BC, Wilce PA. Antibodies against acetaldehydemodified epitopes: an elevated IgA response in alcoholics. Eur J Clin Invest 1991;21:90-5.

Recebido em 24/8/2000 Aprovado em 26/3/2001. 